\title{
A Barnes maze for juvenile rats delineates the emergence of spatial navigation ability
}

\author{
Daniel G. McHail, ${ }^{1,3}$ Nazanin Valibeigi, ${ }^{1,3}$ and Theodore C. Dumas ${ }^{1,2}$ \\ ${ }^{1}$ Krasnow Institute for Advanced Study, George Mason University, Fairfax, Virginia 22030, USA; ${ }^{2}$ Psychology Department, \\ George Mason University, Fairfax, Virginia 22030, USA
}

\begin{abstract}
The neural bases of cognition may be greatly informed by relating temporally defined developmental changes in behavior with concurrent alterations in neural function. A robust improvement in performance in spatial learning and memory tasks occurs at $3 \mathrm{wk}$ of age in rodents. We reported that the developmental increase of spontaneous alternation in a Y-maze was related to changes in temporal dynamics of fast glutamatergic synaptic transmission in the hippocampus. We also showed that, during allothetic behaviors in the Y-maze, network oscillation power increased at frequency bands known to support spatial learning and memory in adults. However, there are no discrete learning and memory phases during free exploration in the Y-maze. Thus, we adapted the Barnes maze for use with juvenile rats. Following a single platform exposure in dim light on the day before training (to encourage exploration), animals were trained on the subsequent $2 \mathrm{~d}$ in bright light to find a hidden escape box and then underwent a memory test $24 \mathrm{~h}$ later. During escape training, the older animals learned the task in $1 \mathrm{~d}$, while the younger animals required $2 \mathrm{~d}$ and did not reach the performance of older animals. Long-term memory performance was also superior in the older animals. Thus, we have validated the use of the Barnes maze for this developmental period and established a timeline for the ontogeny of spatial navigation ability in this maze around $3 \mathrm{wk}$ of age. Subsequent work will pair in vivo recording of hippocampal oscillations and single units with this task to help identify how hippocampal maturation might relate to performance improvements.
\end{abstract}

[Supplemental material is available for this article.]

The hippocampus is part of a forebrain network in mammals that is necessary for spatial cognition (Eichenbaum et al. 2012; Schiller et al. 2015) and serves as a primary model structure for the investigation of the neural bases for changes in learning and memory across the lifespan. In rats, the learning and memory skills needed to perform spatial tasks emerge at 3 wk of age (Douglas et al. 1973; Rauch and Raskin 1984; Rudy et al. 1987; Dumas 2004). This maturation process occurs abruptly and is delayed relative to the developmental onset of numerous other perceptual, motor, and cognitive abilities (Dumas 2005a). For example, Jerry Rudy and colleagues performed numerous experiments in the 1980s and 1990s that clearly illustrated a selective spatial/contextual deficit that persisted to the end of the third postnatal week (cued/spatial water escape task-Rudy et al. 1987; tone/contextual fear conditioningPugh and Rudy 1996; appetitive contextual conditioning-Carew and Rudy 1991). In this vein, more recently used object recognition experiments have shown that at postnatal day (P) 17, rats perform as well as older animals in a novel object-in-context task reliant on salient local cues, but show decreased performance when the task relies on distal cues (Ramsaran et al. 2016a). As such, the late postnatal maturation of spatial learning and memory presents a rare opportunity to identify neural processes that are specifically related to distinct aspects of complex cognitive processes and behaviors.

We previously reported that late postnatal changes in the composition and conductance dynamics of $\alpha$-amino-3-hydroxy5-methyl-4-isoxazolepropionic acid receptors (AMPARs) in the ro-

\footnotetext{
${ }^{3}$ These authors contributed equally to this work.

Corresponding author: tdumas@gmu.edu

Article is online at http://www.learnmem.org/cgi/doi/10.1101//m.046300. 117.
}

dent hippocampus closely paralleled the developmental increase in spontaneous alternation (SA) observed in a Y-maze (Blair et al. 2013). We also reported differences in hippocampal $\theta$ and $\gamma$ oscillations during Y-maze exploration in animals younger and older than 3 wk of age (DG McHail et al. in prep.). However, there is no explicit goal during Y-maze exploration and learning and memory phases cannot be clearly separated. While the Morris water maze (MWM) (Morris 1984) is the gold standard for assessing spatial learning and memory in rodents, the stresses of swimming and ambient temperature introduce confounds for the juvenile animal (Carman and Mactutus 2001; Albani et al. 2014) and the water maze is less compatible with electrophysiological recording.

To overcome these obstacles and contribute a new tool to the study of hippocampal maturation, we adapted a Barnes maze (Barnes 1979) to juvenile rats. Relative to the adult apparatus, the platform size and number of perimeter holes is reduced for juveniles. Animals are first exposed to the platform in dim light and are then trained in bright light on the following $2 \mathrm{~d}$ to locate an escape box hidden beneath one of the platform holes. Escape strategy is tested at the end of each testing day (immediate probe, IP) and $24 \mathrm{~h}$ after the last testing day ( $24 \mathrm{HP}$ ) by removing the escape box and observing the animal's behavior for $1 \mathrm{~min}$. The exploration path for juveniles in dim light covered the platform surface. During escape training in bright lighting, the older animals learned the task in $1 \mathrm{~d}$, while the younger animals required $2 \mathrm{~d}$, but did not

\footnotetext{
(C) 2018 McHail et al. This article is distributed exclusively by Cold Spring Harbor Laboratory Press for the first 12 months after the full-issue publication date (see http://learnmem.cshlp.org/site/misc/terms.xhtml). After 12 months, it is available under a Creative Commons License (Attribution-NonCommercial 4.0 International), as described at http://creativecommons.org/licenses/by-nc/ $4.0 \%$
} 
reach the performance level expressed by the older group. As would be predicted by the enhanced learning, long-term memory performance was also superior in the older animals. Thus, we demonstrated that the Barnes maze can be performed at this developmental stage and can be used to separate differences in cognitive abilities in animals younger and older than $3 \mathrm{wk}$. This work sets the stage for in vivo recording of local field potentials (LFPs) and single units to determine the neural network properties that are responsible for the emergence of spatial learning and memory.

\section{Results}

\section{Habituation in dim light}

At P17 or P22, 1 d prior to training, animals explored the maze platform in dim light for $15 \mathrm{~min}$. Prior to the animal's first movement $(>5 \mathrm{~cm} / \mathrm{sec}$ ), animals paused for a duration that was higher in younger animals (latency $t_{(28.627)}=2.393, P=0.023$ ) (Supplemental Fig. S1A). This might indicate greater hesitation or anxiety in younger animals introduced to a novel environment. The total path length following this initial pause did not differ significantly between age groups $\left(t_{(23.934)}=1.910, P=0.068\right)$ (Supplemental Fig. S1B). When pauses were excluded (when the animal's movement speed was $<5 \mathrm{~cm} / \mathrm{sec}$ ), maximum movement speed during exploration was higher in older animals $\left(t_{(43)}=-2.228, P<0.05\right)$ (Supplemental Fig. S1C), but mean movement speed did not differ between age groups $\left(t_{(43)}=0.867, P=0.391\right)$ (Supplemental Fig. S1D). These data suggest that the older animals were capable of moving faster, but overall maintained a similar rate of movement relative to younger animals. The number of pauses $>1 \mathrm{sec}$ in duration did not vary with age $\left(t_{(43)}=0.575, P=0.568\right)$ (Supplemental Fig. S1E), nor did the average duration of these pauses $\left(t_{(27.381)}=0.000, P=1.000\right)$ (Supplemental Fig. S1F). Taken together, the results suggest that despite a brief initial period of increased hesitation in younger animals, both age groups performed similarly during dim light exploration.

\section{Training Day 1: younger and older animals displayed escape learning}

Analysis of escape latency revealed that both age groups learned to locate the goal after $1 \mathrm{~d}$ of training (RMANOVA main effect for block: $F_{(3.353,164.296)}=51.691, P<0.001$; post hoc tests: $P<0.05$ for block 1 versus 2-5) (Fig. 1A, left). While there was no significant block by age interaction effect $\left(F_{(3.353,164.296)}=1.508, P=0.210\right)$, there was a significant main effect for age $\left(F_{(1,49)}=5.610, P<0.05\right)$, indicating that the older animals outperformed the younger animals (post hoc tests $P<0.05$; blocks 2,4 , and 5). Interestingly, when trials for which the animal did not locate the goal were excluded from the analysis (see Supplemental Table S1), a main effect for block was still present $\left(F_{(2.849,62.271)}=3.305, P<0.05\right.$; post hoc tests: $P<0.05$, block 2 versus 5 ) and the older animals still outperformed younger animals (main effect for age: $F_{(1,22)}=4.741$, $P<0.05$; post hoc: $P<0.01$, block 5) (Fig. 1A, right). This suggests that the overall differences in escape latency were more likely due to animals learning to more frequently locate the goal than to locate the goal more quickly.

Consistent with escape latency, path length also decreased across training blocks (RMANOVA main effect for block: $\left(F_{(4,196)}\right.$ $=22.373, P<0.001$; post hoc tests: $P<0.05$, block 1 versus $3-5$ ) (Fig. 1B, left). There was no block by age interaction effect $\left(F_{(4,196)}=1.020, P=0.398\right)$, but there was a significant main effect for age $\left(F_{(1,49)}=11.167, P<0.01\right)$ where older animals traveled a shorter distance than younger animals during blocks $2-5(P<0.05)$. When trials for which the animal did not locate the goal were excluded from the analysis, there was no longer a significant decrease
A
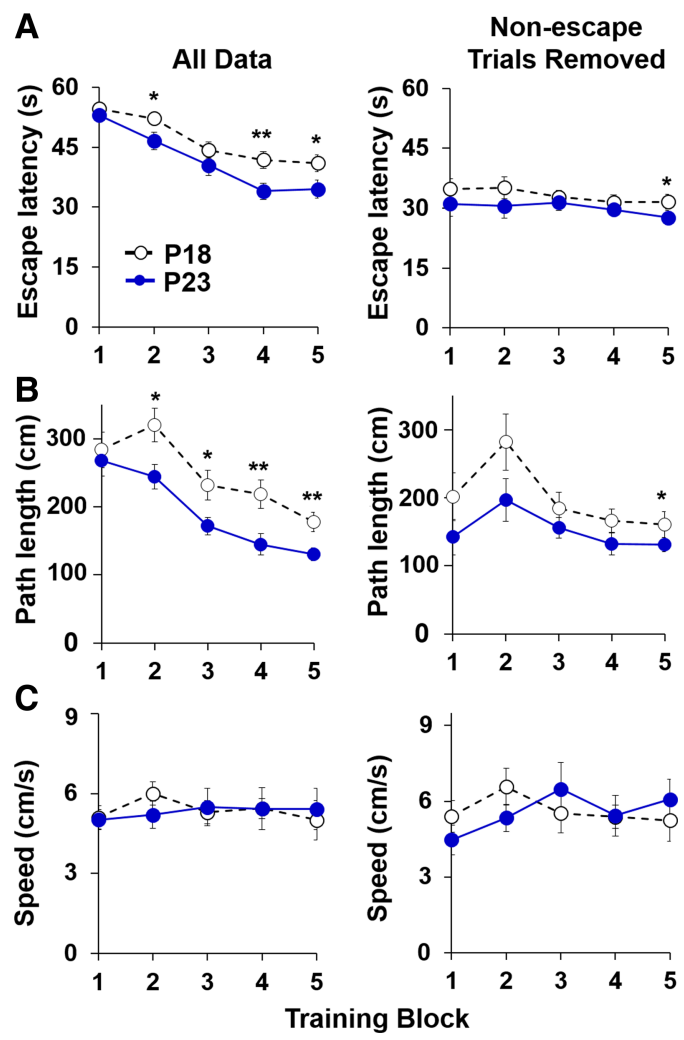

Figure 1. Training Day 1: younger and older animals displayed spatial learning. $(A)$ Mean escape latency per training block for P18 and P23 rats. (B) Mean path length to escape per block. (C) Mean movement speed for escape path. (Left column) Maze performance regardless of whether the goal was entered ("All Data"). (Right column) Maze performance excluding trials for which the goal was not entered ("Non-escape Trials Removed"). Asterisks denote significant pairwise age differences $\left(^{*}\right) P<0.05,\left({ }^{* *}\right) P<0.01$.

in path length across blocks of training (RMANOVA main effect for block $\left.F_{(2.865,60.166)}=2.619, P=0.062\right)$ (Fig. 1B, right). This suggests that the overall decrease in path length across training blocks did not necessarily translate to a more accurate path to the goal. There was no significant block by age interaction $\left(F_{(2.865,60.166)}=0.190, P=0.895\right)$. However, there was a significant main effect for age $\left(F_{(1,21)}=6.321, P<0.05\right)$, where older animals navigated a shorter path to the goal during block $5(P<0.05)$.

In contrast to performance differences in escape latency and path length, there were no significant differences in mean escape speed during training with or without removal of trials for which the animal did not locate the goal (RMANOVA all data: block $F_{(2.966,145.324)}=0.462, P=0.707$; age $F_{(1,49)}=0.006, P=0.939$; block by age interaction $F_{(2.966,145.324)}=0.470, P=0.701$; RMANOVA trials removed: block $F_{(4,80)}=1.977, P=0.106$; age $F_{(1,20)}=0.247$, $P=0.625$; block by age interaction $F_{(4,80)}=0.878, P=0.481$ ) (Fig. $1 \mathrm{C}$, left and right). This suggests that any age differences in escape latency or path length were not due to differences in movement speed. Because the trends in escape latency and path length were both similar, the lack of main effect for block in movement speed is expected.

\section{Training Day 1—first immediate probe: older animals displayed a spatial search strategy}

At the end of Day 1 of training, the escape box was removed and each animal was allowed to search for $60 \mathrm{sec}$. Neither age 
group showed a preference for the goal octant (GOAL) (Fig. 2A). Rather, the majority of time was spent in the opposite (OPP) octant, closest to where the investigator approached the maze to start each training trial (MANOVA main effect for octant: $F_{(2.297,41.354)}=9.161, P<0.001$; post hoc tests: $P<0.05$, OPP versus all octants except GOAL and OPP-1). This octant bias was consistent for both age groups in that there was no main effect of age $\left(F_{(1,18)}=1.091, P=0.310\right)$ or an octant by age interaction $\left(F_{(2.297,41.354)}=1.649, P=0.201\right)$. There was also no age difference when comparing the percent of time in the goal octant relative to the opposite octant $\left(t_{(15.086)}=-0.404, \quad P=0.692\right)$ (Supplemental Fig. S2A). The mean movement speed following initial movement during the probe trial was not significantly different between age groups $\left(t_{(5.347)}=0.031, P=0.977\right.$ ) (Fig. 2B). The maximum movement speed was also not significantly different $\left(t_{(13)}=-1.840, P=0.089\right)$ (Supplemental Fig. S3A), suggesting that performance differences across age groups are not a function of changes in motor ability.
A

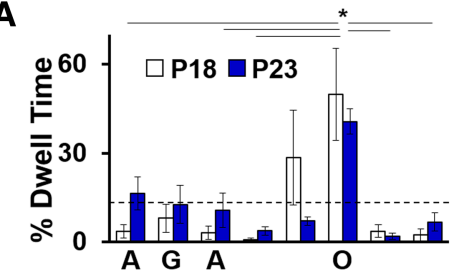

C
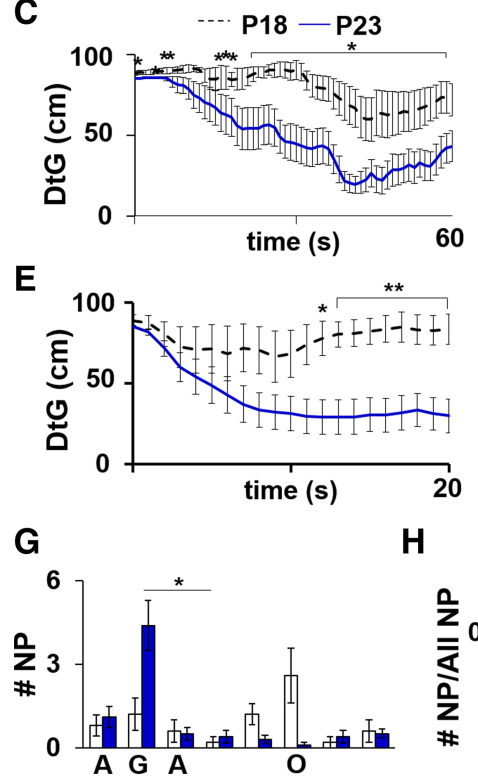

H

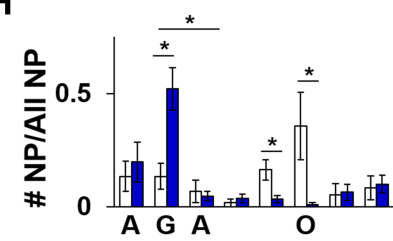

Figure 2. Training Day 1 immediate probe: only the older animals demonstrated recall of goal location. $(A)$ Mean percent dwell time per octant for $\mathrm{P} 18$ and $\mathrm{P} 23$ rats. Octants are shown from left to right relative to clockwise position on maze. (A) adjacent, (G) goal; (O) opposite. Horizontal lines denote significant differences between $O$ and other octants regardless of age. Dashed line denotes chance, $12.5 \%$. (B) Mean movement speed (cm/sec) for younger compared to older animals (C) Raw distance to goal (DtG) per second for younger compared to older animals $(D)$ Mean latency to movement $(>5 \mathrm{~cm} / \mathrm{sec})$ for the $60 \mathrm{sec}$ probe $(E)$ Distance to goal (DtG) starting from initial mobility $>5 \mathrm{~cm} / \mathrm{sec}$. $(F)$ The mean number of error NPs before reaching goal hole. $(G)$ The mean number of NPs per octant during the entire $60 \mathrm{sec}$ probe. Octants are labeled as in $A$. $(H)$ The mean number of NPs per octant normalized by the total number of NPs per trial. In $B-H$, asterisks above horizontal lines denote significant pairwise octant or age comparisons. $\left(^{*}\right) P<0.05,\left({ }^{* *}\right)$ $P<0.01$.
Measures of dwell time are not indicative of actual movement trajectories toward the goal at distinct times during the probe trial. For example, considering both groups together, mean distance between the animal and the goal (DtG) decreased over time $\left(F_{(59,1003)}=7.849, P<0.001\right)$ (Fig. $\left.2 \mathrm{C}\right)$ and there was a main effect of age $\left(F_{(1,17)}=24.799, P<0.001\right)$ with the most pronounced differences occurring between seconds 23-59 (post hoc $P<0.05$ for seconds 23-59 except for seconds 49 and 52). There was also an age by time interaction $\left(F_{(59,1003)}=1.784, P<0.001\right)$, indicating that the decrease in DtG over time was carried primarily by the older animals. Interestingly, animals at both ages similarly paused for an average of 17-32 sec prior to initiating movement (Fig. 2D), suggesting no age difference in initial deliberation or fear. We asked whether maze performance differed between age groups following this pause. Given the variation among animals for the amount of time from first movement to the end of the $60 \mathrm{sec}$ probe, a duration of $20 \mathrm{sec}$ following the initial pause was chosen for the analysis window to maximize the length of data for which most animals could be represented. When calculation began with the initiation of movement, the DtG rapidly decreased in older animals while remaining virtually unchanged in younger animals [main effect for age $\left(F_{(1,14)}=7.682, P<0.05\right)$, main effect for time $\left(F_{(19,266)}=5.108, P<0.001\right)$, time by age interaction $\left.\left(F_{(19,266)}=3.450, P<0.001\right)\right]$ (Fig. $\left.2 \mathrm{E}\right)$. DtG differed across age groups primarily from 13 to $20 \mathrm{sec}$ following initiation of movement $(P<0.01)$ (Fig. 2E). Combined, these results show that the older, but not the younger animals learn to use a spatial search strategy after a single day of training.

Proximity to the goal location as shown by DtG does not directly address whether an animal identified the correct escape hole. Therefore, we measured nose pokes (NPs) in goal and nongoal locations. NPs in nongoal locations prior to the first NP in a goal location were considered errors. There were more than three times as many errors on average in younger compared to older animals $\left(5 \pm 0.8\right.$ versus $\left.1.5 \pm 0.4, t_{(6.816)}=4.520, P<0.01\right)$ (Fig. 2 F). These errors occurred predominantly in the octant opposite the goal in younger animals and the octant adjacent to the goal in older animals (Supplemental Fig. S4A), suggesting qualitatively different search strategies. When these errors were weighted based on distance from goal to establish a goal hole bias, the resulting error score was also higher for younger compared to older animals $\left(t_{(5.536)}=4.375, P<0.01\right)$. These data suggest that total number of error NPs is increased in younger animals and, at this age, the error NPs show no bias toward the goal location. Whereas, a spatial bias is evident even in the error choices at the older testing age.

Considering all NPs (including NPs at the goal hole), there was a significant main effect for octant $\left(F_{(2.610,33.936)}=24.451\right.$, $P<0.01)$. Post hoc analyses were only significant for GOAL versus GOAL+2 $(P<0.05)$. Although there was no main effect for age $\left(F_{(1,13)}=0.025, P=0.877\right)$ there was a significant octant by age interaction $\left(F_{(2.610,33.936)}=6.094, P<0.01\right)$, suggesting that this goal bias was carried by older animals (Fig. 2G). When normalized by the total number of NPs within-animal, results were similar to the distribution of raw NPs except that, relative to younger animals, older animals had a higher number of normalized NPs in the GOAL octant and a lower number of normalized NPs in two octants opposite the goal (main effect for octant $F_{(2.295,29.830)}=$ 4.364, $P<0.05$; post hoc for octant $P<0.05$ only for GOAL versus GOAL+2; main effect for age $F_{(7,7)}=4.293, P<0.05$; post hoc for age $P<0.05$ for GOAL, OPP-1, OPP; octant by age interaction $\left.F_{(2.295,29.830)}=4.759, P<0.05\right)$ (Fig. $\left.2 \mathrm{H}\right)$. These findings suggest that there was a slight goal bias for nose pokes during the first immediate probe for older, but not younger animals.

Overall, the findings show that older animals better learned the spatial location of the goal after $1 \mathrm{~d}$ of training, while younger animals were unable to do so. 
Training Day 2: younger and older animals displayed escape learning

Analysis of escape latency on Day 2 of training revealed a significant main effect for block (RMANOVA main effect for block $F_{(4,196)}=4.515, P<0.01$; post hoc tests; $P<0.05$ for block 1 versus blocks 2-4) (Fig. 3A, left). There was also a significant block by age interaction effect $\left(F_{(4,196)}=3.510, P<0.01\right)$, where the decrease in escape latency was more pronounced in younger animals. However, there was no significant main effect for age $\left(F_{(1,49)}=\right.$ $0.066, P=0.799)$. When trials for which an animal did not enter the goal were excluded from analysis (see Supplemental Table S1), there were no significant differences in escape latency (RMANOVA no main effect for block $F_{(4,184)}=2.285, P=0.062$; no block by age interaction $F_{(4,184)}=2.129, P=0.079$; no main effect for age $F_{(1,46)}=0.409, P=0.526$ ) (Fig. 3A, right). Taken together, these findings suggest that younger animals were more likely to locate the goal with progressive blocks, but training did not impact the speed with which either age group located the goal.

Analysis of path length revealed a significant main effect for block (RMANOVA: $F_{(3.419,164.126)}=9.493, \quad P<0.001$; post hoc tests: $P<0.01$ for blocks 1 versus 2, 4, and 5) (Fig. 3B, left). There was no significant block by age interaction $\left(F_{(3.419,164.126)}=0.892\right.$, $P=0.457)$, suggesting that path length decreased for both age groups. There was a significant main effect for age (RMANOVA: $F_{(1,48)}=21.031, P<0.001$; post hoc tests: $P<0.01$ for blocks 1,2 , 4 , and 5 , suggesting that older animals consistently traveled a shorter distance than younger animals. In contrast to the path
A
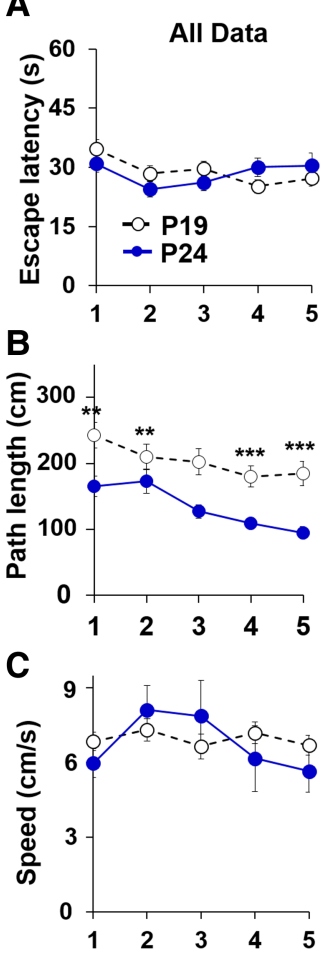

Training Block
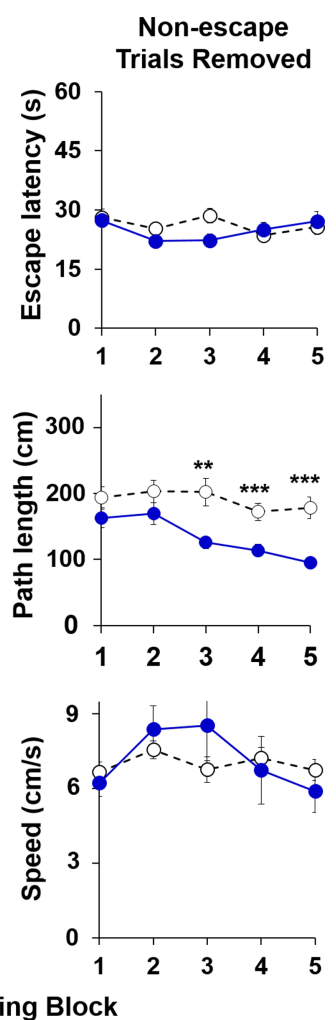

Figure 3. Training Day 2: older animals displayed improved spatial learning. (Left column) Maze performance regardless of whether the goal was entered (Right column) Maze performance excluding trials for which the goal was not entered $(A)$ Mean escape latency per training block for P19 and P24 rats. (B) Mean path length to escape per block. (C) Mean movement speed for escape path. Asterisks denote significant pairwise age differences $\left({ }^{* \star}\right) P<0.01,\left({ }^{* \star *}\right) P<0.001$. length differences on Day 1 of training, differences on Day 2 of training were maintained when trials for which the animal did not enter the goal were excluded (RMANOVA: main effect for block $F_{(3.324,152.912)}=4.993, P<0.01$; post hoc tests: $P<0.05$ for block 1 versus 5,2 versus 4 , and 2 versus 5 ) (Fig. $3 \mathrm{~B}$, right). There was no block by age interaction $\left(F_{(3.324,152.912)}=1.740, P=0.155\right)$. There was a significant main effect for age $\left(F_{(1,46)}=20.580, P<0.001\right)$, where older animals used a shorter path to the goal than younger animals during blocks $3-5$ (post hoc tests: $P<0.01$ ). More specifically, path length to goal was nearly halved in older animals relative to younger animals by block 5 (95 \pm 9 and $179 \pm 16 \mathrm{~cm}$, respectively). This suggests that while some degree of escape learning occurred in the younger animals, older animals were better at solving the task.

Similar to the Day 1 training analysis, mean movement speed on Day 2 did not differ with repeated training or by age (RMANOVA no mean effect for block $F_{(2.792,134.035)}=2.046, P=$ 0.115 ; no block by age interaction $F_{(2.792,134.035)}=1.628, P=0.189$; no main effect for age $F_{(1,48)}=0.032, P=0.858$ ) (Fig. 3C). This finding was robust to removal of trials for which the goal was not entered (RMANOVA no main effect for block $F_{(2.738,125.942)}=2.577$, $P=0.062$; no block by age interaction $F_{(2.738,125.942)}=1.761, P=$ 0.163 ; no main effect for age $F_{(1,46)}=0.066, P=0.798$ ) (Fig. 3C). Given the age differences in path length without corresponding differences in escape latency, lack of main effect for age in movement speed is somewhat surprising. This inconsistency can be explained by the method used for calculating movement speed, where, for each trial, path length was divided by escape latency prior to averaging within block. Thus, group means reported for movement speed are not necessarily similar to the quotient that would be obtained by dividing the group means for path length by escape latency. Interestingly, movement speeds were higher on day 2 compared to day 1 for both age groups (RMANOVA mean effect for day, $F_{(1,48)}=27.385, P<0.001$; no significant interaction effects for day). Taken together, these analyses suggest that both age groups learned to escape on day 2 of training with a developmental improvement in performance level.

\section{Training Day 2-second immediate probe: younger and older animals displayed a spatial search strategy, but older animals outperform younger animals}

A probe trial was performed immediately after training. Similar to the first immediate probe trial, during the second immediate probe trial, there was no main effect of age on octant dwell time $\left(F_{(1,18)}=\right.$ $0.265, P=0.613$ ) (Fig. 4A). There was a goal bias for octant dwell time carried mostly by the older animals (RMANOVA main effect for octant: $\left.F_{(2.419,43.537)}=12.609, P<0.001\right)$ and an octant $\times$ age interaction effect $\left(F_{(2.419,43.537)}=3.455, P<0.05\right)$. Post hoc tests indicated significant dwell time differences between the goal octant and all other octants except the opposite octant and one octant adjacent to the opposite octant $(P<0.05)$. There was a near significant age difference when comparing the percent of time in the goal octant relative to the opposite octant $\left(t_{(17.275)}=-1.778, P=0.092\right)$ (Supplemental Fig. S2B), which might further support a goal bias in older relative to younger animals. The mean movement speed following initial movement during the probe trial was higher in younger animals $\left(t_{(17)}=3.092, P<0.01\right)$ (Fig. $\left.4 \mathrm{~B}\right)$. However, maximum speed was higher for older animals $\left(t_{(17)}=-3.614, P<0.01\right)$ (Supplemental Fig. S3B). This suggests that, while older animals were capable of moving more quickly, younger animals were more active overall.

For DtG during the second immediate probe trial when the timelines were aligned by the start of the trial, there was a main effect for time $\left(F_{(59,1003)}=4.466, P<0.001\right)$. In contrast to the first immediate probe trial, there was no main effect of age 


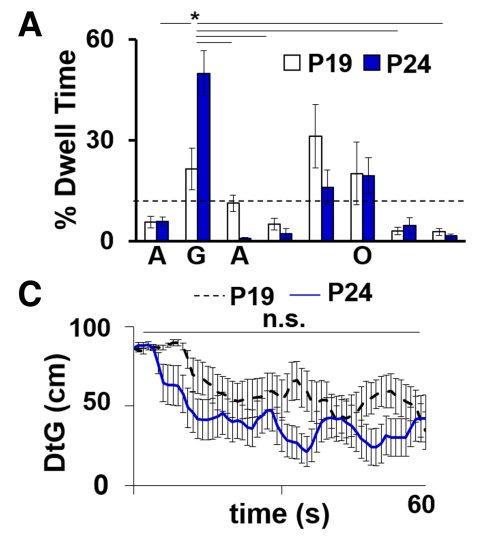

B
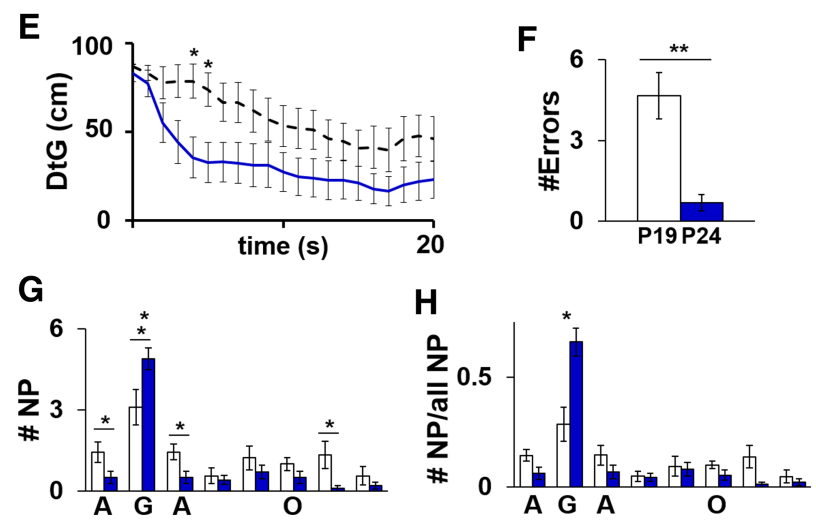

Figure 4. Training Day 2 immediate probe: older animals demonstrated more accurate recall of goal location. $(A)$ Mean percent dwell time per octant for P19 and P24 rats. Octants are shown from left to right relative to clockwise position on maze. (A) adjacent; (G) goal; (O) opposite. Horizontal lines denote significant differences between $O$ and other octants regardless of age. Dashed line denotes chance, $12.5 \%$. (B) Mean movement speed (cm/sec) for younger compared to older animals (C) Raw distance to goal (DtG) per second for younger compared to older animals $(D)$ Mean latency to movement $(>5 \mathrm{~cm} / \mathrm{sec})$ for the $60 \mathrm{sec}$ probe $(E)$ Distance to goal (DtG) starting from initial mobility $>5 \mathrm{~cm} /$ sec. $(F)$ The mean number of error NPs before reaching goal hole. $(G)$ The mean number of NPs per octant during the entire $60 \mathrm{sec}$ probe. Octants are labeled as in $A$. $(H)$ The mean number of NPs per octant normalized by the total number of NPs per trial. In $B-H$, asterisks above horizontal lines denote significant pairwise age comparisons. In $G$ and $H_{\text {, }}$ asterisks without a horizontal line denote significant octant biases relative to all other octants. $\left(^{*}\right) P<0.05,\left(^{* *}\right) P<0.01$.

$\left(F_{(1,17)}=3.899, P=0.065\right)$ or an age by time interaction $\left(F_{(59,1003)}=\right.$ $0.669, P=0.974$ ) (Fig. 4 C). There was no age difference in pause duration prior to initial movement $\left(t_{(13.006)}=1.198, P=0.252\right)$ (Fig. 4D), again suggesting no age difference in initial deliberation or fear. When starting the analysis from onset of movement, DtG decreased over time $\left(F_{(19,304)}=8.748, P<0.001\right)$ with no age by time interaction $\left(F_{(19,304)}=0.688, P=0.831\right)$ (Fig. $\left.4 \mathrm{E}\right)$. However, a main effect for age was found $\left(F_{(1,16)}=4.877, P<0.05\right)$ with greater proximity to goal observed for older animals being statistically significant during seconds $5-6(P<0.05)$. However, visually, the curves are very different for almost the entire timeline. These data suggest that both age groups could navigate toward the goal location, but older animals were still superior, especially during initial orientation.

As during the Training Day 1 probe trial, during the Training Day 2 probe trial, younger animals made several fold more error NPs than older animals $\left(t_{(9.908)}=4.328, P<0.01\right)$ (Fig. $4 \mathrm{~F}$ ). The distribution of these errors is shown in Supplemental Figure
S4B. Considering all NPs (including NPs at the goal hole), there was a significant goal bias (main effect for octant, $F_{(3.482,59.196)}=$ $25.679, P<0.001$ ) carried mostly by the older animals (main effect for age, $F_{(1,17)}=5.269, \quad P<0.05 ; \quad$ octant $\times$ age interaction, $\left.F_{(3.482,59.196)}=4.093, P<0.01\right)$ (Fig. 4G). Post hoc tests indicated more NPs for the GOAL than any other octant $(P<0.001)$. Pairwise comparisons showed a greater number of NPs at the goal hole for older compared to younger animals $(P<0.05)$ and fewer NPs for older compared to younger animals in GOAL-1, GOAL +1 , and OPP $+1(P<0.05)$. This suggests that while older animals showed a strong goal preference with NPs, younger animals demonstrated a more generalized memory of goal location that was distributed among the goal and the two holes adjacent to the goal. When normalized by the total number of NPs within-animal, there was still a main effect of octant $\left(F_{(2.857,45.710)}=26.141, P<0.001\right)$, but no longer a main effect of age weighted NPs $\left(F_{(1,16)}=1.663\right.$, $P=0.225)$. Again, the goal bias was carried mostly by older animals (octant by age interaction effect, $F_{(2.857,45.710)}=7.872, P<0.001$ ) (Fig. $4 \mathrm{H}$ ). These findings suggest that the number of choices per animal likely contributed to the overall age differences found in the raw NPs measure. However, older animals continued to display more accurate spatial search strategy at the end of the second day of training. Generally, age differences in performance were reduced during the Training Day 2 probe relative to the Training Day 1 probe as younger animals started to catch up to the older animals.

\section{Memory probe: spatial memory was more accurate in the older animals}

Twenty-four hours after the final training block, the escape box was removed and a final probe trial (24HP) was performed to assess long-term memory for the goal location. When considering the percent dwell time per octant, there was a significant goal bias (main effect for octant, $\left.F_{(3.803,171.127)}=24.062, P<0.001\right)$ and post hoc tests revealed greater time in the goal octant than any other octant $(P<0.001)$, but there was no main effect of age $\left(F_{(1,45)}=0.838, P=\right.$ 0.365 ) (Fig. 5A). However, an age by octant interaction effect $\left(F_{(3.803,171.127)}=3.770, P<0.01\right)$ supported more time spent in octants near the goal location for the older animals. Further reinforcing a goal bias in older relative to younger animals, percent dwell time was higher in older animals comparing the difference between percent of time in the goal octant relative to the opposite octant $\left(t_{(45)}=-2.444, P=0.019\right)$ (Supplemental Fig. S2C). Similar to the second immediate probe trial, younger animals displayed a higher mean movement speed than older animals $\left(t_{(46)}=3.014, P<0.01\right)$ (Fig. 5B). However, the maximum speed was not different between age groups $\left(t_{(46)}=-1.187, P=0.241\right)$ (Supplemental Fig. S3C). This suggests that, overall, younger animals were more active than older animals during their search for the goal.

When aligned by the start of the trial, DtP measures revealed a main effect for time (RMANOVA $F_{(59,2655)}=5.731, P<0.001$ ) with no time by age interaction $\left(F_{(59,2655)}=0.662, P=0.978\right)$ (Fig. $5 C)$. There was a significant main effect for age $\left(F_{(1,45)}=5.783, P\right.$ $<0.05$ ). However, post hoc tests were only $P<0.05$ for seconds 29-34, so this age difference is not especially meaningful. There was no significant difference in latency to movement between age groups for the $24 \mathrm{HP}\left(t_{(34.921)}=-1.249, P=0.220\right)$ (Fig. 5D). See Supplemental Figure S5C for age-dependent relationships of the length of this pause to other metrics of maze performance. When analysis was started at initial movement for each group, animals approached the goal regardless of age (main effect for time, $F_{(19,855)}=15.803, P<0.001 ;$ no time by age interaction effect, $\left.F_{(19,855)}=1.399, P=0.118\right)$. However, reduced DtP measures indicated that older animals consistently outperformed younger animals (main effect for age, $F_{(1,45)}=14.123, P<0.001 ; P<0.05$ for seconds 3-20) (Fig. 5E). 
A

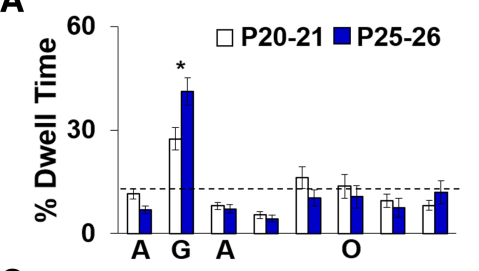

C
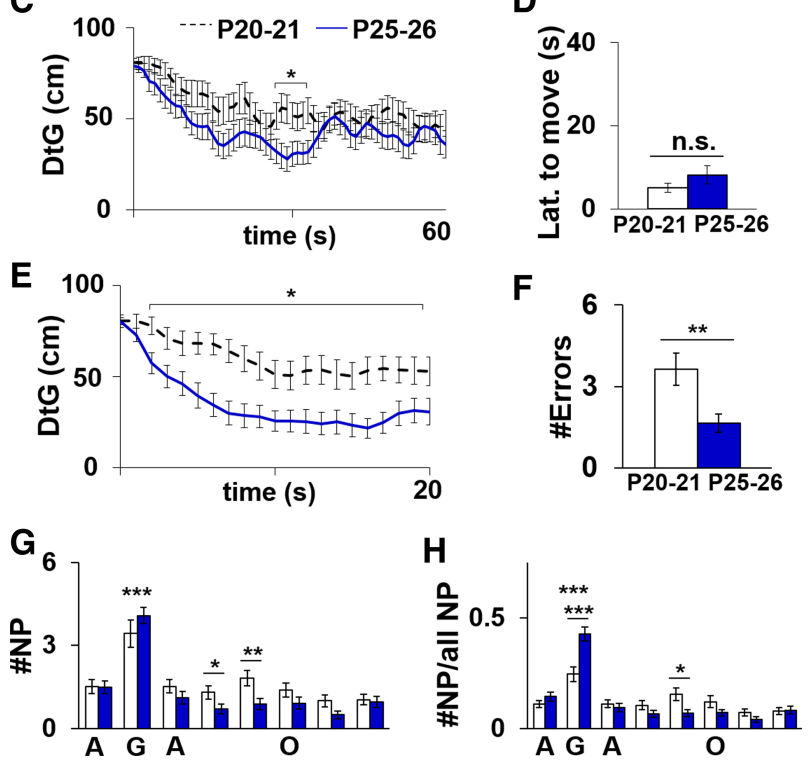

Figure 5. Older animals demonstrated more accurate long-term spatial memory during 24HP. (A) Mean percent dwell time per octant for P20 and P25 rats. Octants are shown from left to right relative to clockwise position on maze. (A) adjacent; (G) goal; (O) opposite. Dashed line denotes chance, $12.5 \%$. (B) Mean movement speed (cm/sec) for younger compared to older animals (C) Raw distance to goal (DtG) per second for younger compared to older animals $(D)$ Mean latency to movement $(>5$ $\mathrm{cm} / \mathrm{sec}$ ) for the $60 \mathrm{sec}$ probe $(E)$ Distance to goal (DtG) starting from initial mobility $>5 \mathrm{~cm} / \mathrm{sec}$. $(F)$ The mean number of error NPs before reaching goal hole. $(G)$ The mean number of NPs per octant during the entire $60 \mathrm{sec}$ probe. Octants are labeled as in $A$. $(H)$ The mean number of NPs per octant normalized by the total number of NPs per trial. In $B-H$, asterisks above horizontal lines denote significant pairwise age comparisons. In $G$ and $H$, asterisks without a horizontal line denote significant octant biases relative to all other octants. $\left(^{*}\right) P<0.05$, (**) $P<0.01$, $\left(^{* * *}\right) P<$ 0.001 .

As in both immediate probes, younger animals made more nose poke errors than older animals prior to locating the goal $\left(t_{(38.373)}=2.929, P<0.01\right)$ (Fig. 5F). The distribution of errors among octants is shown in Supplemental Figure S4C. Considering all NPs per octant throughout the $24 \mathrm{HP}$, there was a significant goal bias (MANOVA main effect for octant: $F_{(3.783,170.213)}=29.309, P<$ 0.001; post hoc tests for octant: $P<0.001$ for goal versus all other octants, $P<0.01$ for GOAL-1 versus OPP +1 ) and a main effect of age $\left(F_{(1,45)}=4.908, P<0.05\right.$; post hoc tests: $P<0.05$ for GOAL +2 and OPP-1) (Fig. 5G). There was no time $\times$ age interaction $\left(F_{(3.783,170.213)}=1.869, P=0.122\right)$. Therefore, while younger animals made more errors than older animals prior to locating the goal, animals in both age groups equally demonstrated recall of the goal location throughout the $24 \mathrm{HP}$. However, when the number of NPs per octant was normalized to each animal's total NPs per trial, the goal bias was carried predominantly by older animals (MANOVA main effect for octant, $F_{(3.922,176.501)}=31.011$, $P<0.001$; octant by age interaction effect, $F_{(3.922,176.501)}=6.635$, $P<0.001$; post hoc tests for octant $P<0.001$ for goal versus all octants and $P<0.01$ for GOAL-1 versus $\mathrm{OPP}+1$; main effect for age $F_{(1,45)}=2.287, P<0.05$; post hoc tests for age significant for goal, $P<0.001$, and OPP-1, $P<0.05$ ) (Fig. $5 \mathrm{H}$ ). Specifically, while nearly half $(0.43 \pm 0.03)$ of each older animal's NPs were in the goal location on average, roughly a quarter of the younger animals' NPs were in the goal location $(0.25 \pm 0.03)$. This suggests a greater accuracy for recall of the goal location in the older age group relative to younger animals.

\section{No sex differences during training or memory probes}

There were no significant main effects of sex or interaction between sex and any other variable (e.g., age, time, and octant) during training (escape latency, path length, movement speed with or without nonescape trials removed) or probe trials (dwell time, distance to goal, distance to goal after initial movement, nose pokes, or normalized nose pokes). Therefore, data were collapsed across sex for all analyses reported above.

\section{Discussion}

The results presented in this report validate the Barnes maze as an appropriate behavioral tool to probe the development of spatial learning and memory in rats. Animals of both age groups learned to find the escape hole during training. However, older animals applied a spatial search strategy on the first day of training, while younger animals did not show signs of spatial navigation until the second day of training. The search strategy used by older animals during the immediate and long-term memory probes was consistently more direct and accurate than that used by younger animals. Thus, we have identified several points where maze performance contrasts sharply depending on age, and each of these are potential targets for further investigation into the maturation of neural substrates for these behaviors.

Our findings in this report align well with prior work in rats that showed the emergence of spatial navigation ability at P21, including SA in a Y-maze (Douglas et al. 1973; Dumas 2004), learning and memory in the MWM (Rudy et al. 1987; Carman and Mactutus 2001), and contextual fear conditioning (Pugh and Rudy 1996; Travaglia et al. 2016). However, while also reliant on an intact hippocampus (Langston and Wood 2010; Warburton and Brown 2015), object-place conditioning and object-place-context memory abilities are not evident until the animals are more than a month old (Ramsaran et al. 2016b). Thus, full maturation of the hippocampus or hippocampal formation (including the entorhinal cortex and subiculum) may be more protracted, with more complex, episodic-like abilities requiring greater time to mature than more basic spatial skills (Tan et al. 2016).

While multimodal sensory input contributes to spatial navigation, the Barnes maze is primarily a visual task. Adult rodents have been shown to preferentially rely on distal, extramaze cues even when intramaze visual cues are present (Harrison et al. 2006). The maze we describe lacks intra-maze visual cues, and start locations are randomized to encourage a spatial search strategy. Olfactory cues, however, are a potential confound, as salient olfactory cues can potentiate spatial learning in adult rodents (Lavenex and Schenck 1997) and have been shown to support place cell activity (Kulvicius et al. 2008). In addition, rodents have been shown to follow their own odor trails and the odor trails of conspecifics (Wallace et al. 2002). It is recommended to rotate the maze in between trials relative to a fixed goal location to ensure that odor trails do not lead to the goal (Barnes 1979). Wiping the maze with $70 \%$ ethanol in between maze exposures is also recommended to minimize olfactory cues (Rosenfeld and Ferguson 2014), but it is possible that the MWM might more effectively control for olfactory cues (Morris 1984). When visual extramaze cues are present as 
well as olfactory cues, adult rats preferentially use a spatial strategy (Olton and Collison 1979; Lavenex and Schenck 1995; Maaswinkel and Whishaw 1999).

Less is known about the hierarchy of senses that directs spatial navigation in immature animals. One report shows a preference for salient olfactory cues over visual cues in P24 rats in a homing board task, while this preference is eliminated in 3-6-month old rodents and reversed in aged (12-month old) rats (Rossier and Schenk 2003). While the Barnes maze task in this report lacks a salient olfactory cue and scent trails are removed with ethanol between different animals and after each training block, it might be informative to investigate whether any residual odors influence maze performance across the P17-25 developmental trajectory. Since olfaction in rodents reaches adult levels of discrimination before P17 (Gregory and Pfaff 1971; Cornwell-Jones and Sobrian 1977), changes in olfactory capabilities from P17-25 are unlikely to account for the age differences in Barnes maze performance. Auditory and visual senses continue to mature across this period (Crowley and Hepp-Reymond 1966; Hyson and Rudy 1984; Fagiolini et al. 1994; Morishita and Hensch 2008; Tan et al. 2016), but are unlikely to account for age differences in the Barnes maze as potential auditory cues were masked by the overhead ventilation system and visual cues were enlarged to accommodate juvenile animal eyesight (Albani et al. 2014). It is, therefore, unlikely that maturation of perceptual abilities influenced age differences reported in the Barnes maze in juvenile rats, and that deficits we report in rats under P21 are predominantly spatial.

Further supporting the idea that age differences in the Barnes maze are due to maturation of navigation ability is the lack of changes in other abilities reported in this study and in other reports on this developmental period. Average movement speed during both days of training and IP1 did not differ between age groups, and maximum movement speed did not vary with age for IP1 and $24 \mathrm{HP}$, suggesting that locomotor ability was equivalent between age groups for these trials. Since average movement speed following initial movement was higher in younger animals during IP2 and 24HP without a concurrent increase in maximum movement speed (in fact, maximum movement speed was higher in older animals during IP2), this increase in younger animals is likely due to hyperactivity in search strategy rather than a difference in locomotor ability. Mature locomotor ability on a rough surface has previously been demonstrated in rats as early as P16, although with a smooth surface asymptotic levels of locomotion were not observed until P19-20 (Altman and Sudarshan 1975). The current Barnes maze surface is smooth, but a rougher surface might be used in future studies to further minimize age differences in locomotion. Age differences in the Barnes maze are also not likely due to differences in anxiety, as animals at both ages spent an equal amount of time deliberating before initial movement. Consistent with this, rats at P17-19 and P22-24 spent equal time in the open (exposed) arm of an elevated plus maze (EPM) in a prior study (Albani et al. 2015). Finally, we showed that there were no sex differences in Barnes maze performance for either age group. Although sex differences have been reported in adult rats in the MWM (Roof and Stein 1999), lack of sex differences in juvenile rats in the Barnes maze is not surprising given that rats are prepubertal until around P30 in females and P35 in males (Ojeda and Urbanski 1994). Previous studies of prepubertal rats found no sex differences in contextual fear conditioning (Beane et al. 2002) or object-context or object-place-context discrimination tasks (Ramsaran et al. 2016b), supporting that sex differences in sensitivity to spatial context are not evident before puberty. Thus, age differences in the Barnes maze are likely spatial and not due to locomotion, anxiety, or sexual maturation.

Developmental alterations in hippocampal baseline excitatory synaptic transmission and synaptic plasticity occur in parallel with the onset of spatial navigation ability (Dumas 2005a,b, 2012; Stoneham et al. 2010). Near the end of the third postnatal week, a change in the subunit composition of hippocampal AMPA receptors has been linked to a prolonged AMPAR response, which enhances synaptically driven postsynaptic discharge and lowers the threshold for induction of long-term potentiation (LTP). Pharmacological prolongation of AMPAR responses by AMPAkines (Arai and Kessler 2007) in immature rats increased SA in a Y-maze and mimicked the effects of development by increasing postsynaptic discharge and reducing the threshold for induction of LTP, suggesting that mature AMPAR function is sufficient to enable rudimentary spatial navigation ability (Blair et al. 2013). AMPAkine administration to immature rats prior to Barnes maze testing might further clarify the role of AMPAR maturation in distinct aspects of spatial cognition (i.e., learning, memory maintenance, and memory retrieval).

Late postnatal modifications at the network level are also evident in the hippocampal formation. Longitudinal recordings of LFPs in the behaving rat hippocampus have revealed increased $\theta$ power during random foraging in a cylinder (Langston et al. 2010; Wills et al. 2010) and increased $\theta$ peak frequency during exploration in a Y-maze (DG McHail et al. in prep.). At the single unit level, head direction cells in the pre- and parasubiculum and medial entorhinal cortex appear mature prior to the end of the third postnatal week (Bjerknes et al. 2015), while place cells and grid cells continue to develop beyond 3 wk of age (Langston et al. 2010; Bjerknes et al. 2014; Muessig et al. 2015; for a recent review, see Tan et al. 2016). These developmental studies of hippocampal network dynamics in juvenile rats have not yet been paired with a goal-directed task having discrete learning and memory phases. We propose the Barnes maze as a goal directed task that resolves developmental differences in spatial cognition and is compatible with in vivo hippocampal single unit and LFP recording.

In addition to providing a tool to help decode the neural underpinnings of spatial navigation, this report also establishes a timeline for the emergence of spatial navigation ability in neurotypical juveniles in the Barnes maze task. This baseline will be useful in animal models of neurodevelopmental disorders associated with impaired cognition. For example, deficits in spatial cognition have been observed in rodent models of autism (Ellegood and Crawley 2015). Additionally, this new approach helps to build a lifespan approach to better understand memory impairment in aging through the collection of behavioral performance data from juveniles that can be more directly compared to a large body of data collected in aged rodents.

\section{Materials and Methods}

\section{Subjects}

Male and female Long Evans rats used for this study were bred in the Krasnow Institute Animal Facility (accredited by the Association for Assessment and Accreditation of Laboratory Animal Care International, AAALAC). Original and replacement breeders were purchased from Charles River Laboratories. Animals were maintained in individually ventilated cages (Animal Care Systems) on a 12:12 h light-dark cycle with lights coming on at 8 a.m. Water and food (Envigo's Teklad LM-485 7012) were available ad libitum. All procedures were performed in accordance with the regulations stated in the Guide for Care and Use of Laboratory Animals by the National Research Council and approved by the George Mason University Institutional Animal Care and Use Committee.

\section{Behavior testing}

The adapted Barnes maze consisted of a white, circular platform (diameter $1.2 \mathrm{~m}$ ) elevated $0.75 \mathrm{~m}$ from the ground with eight evenly spaced holes (diameter $10 \mathrm{~cm}$ ) $5 \mathrm{~cm}$ from the maze perimeter 
(Fig. 6A). An escape box was positioned under one of the escape holes, designated the goal location, for the entirety of training and memory testing. Goal location was counterbalanced among litters but remained consistent for both age groups within a litter. The maze was positioned in the center of the testing room $(3.6 \times$ $5.25 \mathrm{~m}$ ). Two floor lamps positioned on either side of the maze and cloth curtains patterned with large, geometric shapes (circles, triangles, and stripes) served as extramaze cues. An overhead camera (Model WV-CP280-N, 1/3" CCD, Panasonic, 30 frames/sec) and video software (ULead Video Studio, Corel) recorded animal behavior during each trial.

A total of 51 animals from 5 different litters (27 males, 24 females) were tested in the maze. Roughly half of the animals in each litter were tested at P17-20, while the other half was tested at P2225. One litter was tested a day late (P18-21 and P23-26). The animal's home cage with dam and littermates was brought to a biosafety cabinet in the behavior room during testing and each animal was returned to the home cage immediately following each maze exposure or training block. The maze was wiped with $70 \%$ ethanol between animals and prior to the first animal's maze exposure. The room's ventilation system provided a soft white noise background.

Each cohort was tested for four consecutive days according to the schedule outlined in Figure 6B. While the MWM forces animals to swim, on the Barnes maze there is no impetus to move. Therefore, on the first day of testing, each animal was placed in the center of the maze in dim light (floor lamps at low setting, no overhead light; 25 lux) and allowed to freely explore for $15 \mathrm{~min}$ to become familiar with the maze and escape holes. No escape box was present for this initial maze exposure. On the following day, the escape box was positioned under one of the escape holes and floor lamps and overhead lights were turned on to their maximum brightness ( 525 lux). Each animal was introduced to the maze at one of four pseudorandomly ordered start locations positioned in between two escape holes and allowed $60 \mathrm{sec}$ to locate the escape box. If an animal did not find the escape box, it was gently led to the goal by the experimenter. Five training blocks consisting of four trials were administered on each day for $2 \mathrm{~d}$ of

A
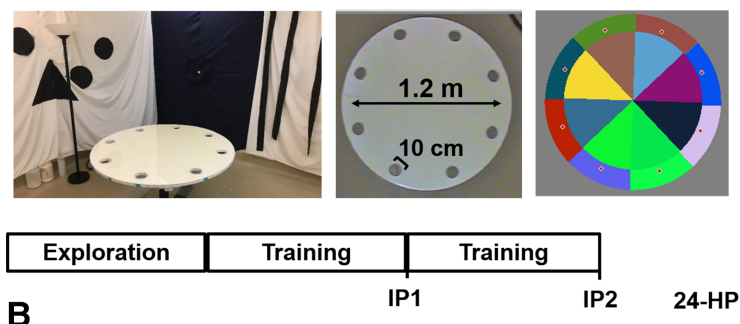

B

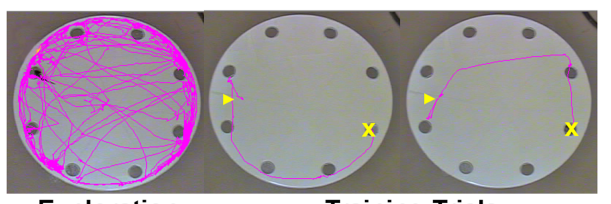

Exploration

Training Trials

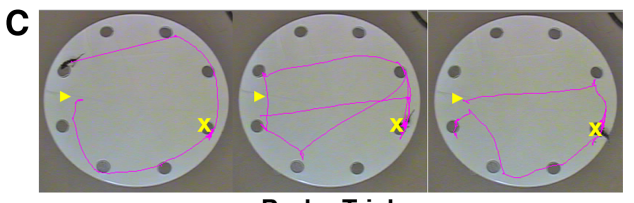

Probe Trials

Figure 6. A Barnes maze protocol was developed for juvenile rats. $(A)$ Photographs of maze and visual cues (left), maze dimensions (middle), and regions used for analysis (right). (B) Testing schedule. (IP) immediate probe; (24HP) $24 \mathrm{~h}$ probe. (C) Illustrations of typical paths for an animal tested at P22-25. (Top) Paths during exploration and late in training. (Bottom) Probe trials. Arrowhead: start location; " $\mathrm{X}$ ": goal location. training with an interblock interval of 15-30 min. Twenty-four hours following the last training block, the escape box was removed and each animal was allowed 60 sec to search for the goal (24HP). A subset of animals (2 litters, 10 animals per age group), also received an immediate probe (IP) following the fifth training block of each day. For these animals, the escape box was returned to the goal location for a refresher block following the IP on the second training day. Figure $6 \mathrm{C}$ shows typical navigation paths for each day of the behavioral training procedure.

\section{Behavior analysis}

Paths during free exploration on the day prior to training were not analyzed for this report. For analysis of maze performance during training, escape latencies were recorded by stopwatch and path length was analyzed offline using behavior tracking software (Topscan Version 2.00, CleverSys). All four paws were required to enter the escape box for a successful goal entry. For analysis of probe trials (IP1, IP2, and 24HP), octant dwell time, movement speed, distance from the goal center (DtG) analyzed at $1 \mathrm{~Hz}$, latency to first movement, and number and position of nose pokes into holes were extracted from the overhead videos. For latency to movement measurements, initiation of movement was considered when the movement speed reached $5 \mathrm{~cm} / \mathrm{sec}$. For analysis of nose pokes (NPs), a separate nose poke was counted each time the animal's head entered the hole as seen from above.

\section{Statistics}

Student's $t$-test, repeated-measures ANOVA (RMANOVA), multivariate ANOVA (MANOVA), and linear regression were conducted in SPSS (IBM SPSS Statistics). Two-tailed, independent $t$-tests were used to compare means for movement speed, latency to movement, and the number of nose poke errors between age groups. Where Levene's test for homogeneity of variances was significant, degrees of freedom and $P$-values were adjusted accordingly. RMANOVA was used to compare (1) mean escape latency, path length to goal, and movement speed during navigation to goal between age groups over blocks of training, (2) percent dwell time and mean number of nose pokes between age groups over individual octants, and (3) mean distance to goal between age groups over time during probe trials. Where Mauchly's test indicated that the assumption of sphericity was not met, the Greenhouse-Geisser correction was applied. When a main effect for age or interaction effect was significant, MANOVA was used as a follow-up test to compute pairwise differences between age groups. Bonferroni's correction was used for all post hoc comparisons. Linear regression was used to relate latency to movement to maze performance. For all tests, $P<0.05$ was considered statistically significant. Data points and error bars shown in all figures are mean \pm SEM.

\section{Acknowledgments}

The authors thank Amir Behsudi and Celeste Chong for their technical support. This work was supported by the National Institutes of Health (NIH) National Institute on Aging (NIA) through grant 1R15AG045820-01A1 and the Krasnow Institute for Advanced Study at George Mason University. D.G.M. is supported by a DoD SMART Scholarship.

ReferencesAlbani SH, McHail DG, Dumas TC. 2014. Developmental studies of the hippocampus and hippocampal-dependent behaviors: insights from interdisciplinary studies and tips for new investigators. Neurosci Biobehav Rev 43: 183-190.

Albani SH, Andrawis MM, Abella RJH, Fulghum JT, Vafamand N, Dumas TC. 2015. Behavior in the elevated plus maze is differentially affected by testing conditions in rats under and over three weeks of age. Front Behav Neurosci 9: 31.

Altman J, Sudarshan K. 1975. Postnatal development of locomotion in the laboratory rat. Anim Behav 23: 896-920.

Arai AC, Kessler M. 2007. Pharmacology of ampakine modulators: from AMPA receptors to synapses and behavior. Curr Drug Targets 8: 583-602. 
Barnes CA. 1979. Memory deficits associated with senescence: a neurophysiological and behavioral study in the rat. J Comp Physiol Psychol 93: 74-104.

Beane ML, Cole MA, Spencer RL, Rudy JW. 2002. Neonatal handling enhances contextual fear conditioning and alters corticosterone stress responses in young rats. Horm Behav 41: 33-40.

Bjerknes TL, Moser EI, Moser MB. 2014. Representation of geometric borders in the developing rat. Neuron 82: 71-78.

Bjerknes TL, Langston RF, Kruge IU, Moser EI, Moser MB. 2015. Coherence among head direction cells before eye opening in rat pups. Curr Biol 25: 103-108.

Blair MG, Nguyen NN, Albani SH, L'Etoile MM, Andrawis MM, Owen LM, Oliveira RF, Johnson MW, Purvis DL, Sanders EM, Stoneham ET, Xu H, Dumas TC. 2013. Developmental changes in structural and functional properties of hippocampal AMPARs parallels the emergence of deliberative spatial navigation in juvenile rats. J Neurosci 33: $12218-12228$.

Carew MB, Rudy JW. 1991. Multiple functions of context during conditioning: a developmental analysis. Dev Psychobiol 24: 191-209.

Carman HM, Mactutus CF. 2001. Ontogeny of spatial navigation in rats: a role for response requirements? Behav Neurosci 115: 870-879.

Cornwell-Jones C, Sobrian SK. 1977. Development of odor-guided behavior in Wistar and Sprague-Dawley rat pups. Physiol Behav 19: 685-688.

Crowley DE, Hepp-Reymond M-C. 1966. Development of cochlear function in the ear of the infant rat. J Comp Physiol Psych 62: 427-432.

Douglas RJ, Peterson JJ, Douglas DP. 1973. The ontogeny of a hippocampusdependent response in two rodent species. Behav Biol 8: 27-37.

Dumas TC. 2004. Early eyelid opening enhances spontaneous alternation and accelerates the development of perforant path synaptic strength in the hippocampus of juvenile rats. Dev Psychobiol 45: 1-9.

Dumas TC. 2005a. Late postnatal maturation of excitatory synaptic transmission permits adult-like expression of hippocampal-dependent behaviors. Hippocampus 15: 562-578.

Dumas TC. 2005b. Developmental regulation of cognitive abilities: modified composition of a molecular switch turns on associative learning. Prog Neurobiol 76: 189-211.

Dumas TC. 2012. Postnatal alterations in induction threshold and expression magnitude of long-term potentiation and long-term depression at hippocampal synapses. Hippocampus 22: 188-199.

Eichenbaum H, Sauvage M, Fortin N, Komorowski R, Lipton P. 2012. Towards a functional organization of episodic memory in the medial temporal lobe. Neurosci Biobehav Rev 36: 1597-1608.

Ellegood J, Crawley JN. 2015. Behavioral and neuroanatomical phenotypes in mouse models of autism. Neurotherapeutics 12: 521-533.

Fagiolini M, Pizzorusso T, Berardi N, Domenici L, Maffei L. 1994. Functional postnatal development of the rat primary visual cortex and the role of visual experience: dark rearing and monocular deprivation. Vision Res 34: 709-720.

Gregory EH, Pfaff DW. 1971. Development of olfactory-guided behavior in infant rats. Physiol Behav 6: 573-576.

Harrison FE, Reiserer RS, Tomarken AJ, McDonald MP. 2006. Spatial and nonspatial escape strategies in the Barnes maze. Learn Mem 13: 809-819.

Hyson RL, Rudy JW. 1984. Ontogenesis of learning: II. Variation in the rat's reflexive and learned responses to acoustic stimulation. Dev Psychobiol 17: $263-283$.

Kulvicius T, Tamosiunaite M, Ainge J, Dudchenko P, Wörgötter F. 2008. Odor supported place cell model and goal navigation in rodents. J Comput Neurosci 25: 481-500.

Langston RF, Wood ER. 2010. Associative recognition and the hippocampus: differential effects of hippocampal lesions on objectplace, object-context and object-place-context memory. Hippocampus 20: $1139-1153$.

Langston RF, Ainge JA, Couey JJ, Canto CB, Bjerknes TL, Witter MP, Moser EI, Moser M-B. 2010. Development of the spatial representation system in the rat. Science 328: 1576-1580.
Lavenex P, Schenck F. 1995. Influence of local environmental olfactory cues on place learning in rats. Physiol Behav 58: 1059-1066.

Lavenex P, Schenck F. 1997. Olfactory cues potentiate learning of distant visuospatial information. Neurobiol Learn Mem 68: 140-153.

Maaswinkel H, Whishaw IQ. 1999. Homing with locale, taxon, and dead reckoning strategies by foraging rats: sensory hierarchy in spatial navigation. Behav Brain Res 99: 143-152.

Morishita H, Hensch TK. 2008. Critical period revisited: impact on vision. Curr Opin Neurobiol 18: 101-107.

Morris R. 1984. Developments of a water-maze procedure for studying spatial learning in the rat. J Neurosci Methods 11: 47-107.

Muessig L, Hauser J, Wills TJ, Cacucci F. 2015. A Developmental Switch in Place Cell Accuracy Coincides with Grid Cell Maturation. Neuron 86: 1167-1173.

Ojeda SR, Urbanski HF. 1994. Puberty in the rat. In The Physiology of Reproduction, 2nd ed. (ed. Knobil E, Neill JD), pp. 363-409. Raven Press, Ltd., New York.

Olton DS, Collison C. 1979. Intramaze cues and odor trails fail to direct choice behavior on an elevated maze. Anim Learn Behav 7: 221-223.

Pugh CR, Rudy JW. 1996. A developmental analysis of contextual fear conditioning. Dev Psychobiol 29: 87-100.

Ramsaran AI, Westbrook SR, Stanton ME. 2016a. Ontogeny of object-incontext recognition in the rat. Behav Brain Res 298: 37-47.

Ramsaran AI, Sanders HR, Stanton ME. 2016b. Determinants of object-incontext and object-place-context recognition in the developing rat. Dev Psychobiol 58: 883-895.

Rauch SL, Raskin LA. 1984. Cholinergic mediation of spatial memory in the preweanling rat: Application of the radial arm maze paradigm. Behav Neurosci 98: 35-43.

Roof RL, Stein DG. 1999. Gender differences in Morris water maze performance depend on task parameters. Physiol Behav 68: 81-86.

Rosenfeld CS, Ferguson SA. 2014. Barnes maze testing strategies with small and large rodent models. J Vis Exp 84: e51194.

Rossier J, Schenk F. 2003. Olfactory and/or visual cues for spatial navigation through ontogeny: olfactory cues enable the use of visual cues. Behav Neurosci 117: 412-425.

Rudy JW, Stadler-Morris S, Albert P. 1987. Ontogeny of spatial navigation behaviors in the rat: dissociation of "proximal" and "distal" - cue-based behaviors. Behav Neurosci 101: 62-73.

Schiller D, Eichenbaum H, Buffalo EA, Davachi L, Foster DJ, Leutgeb S, Ranganath C. 2015. Memory and space: towards an understanding of the cognitive map. J Neurosci 35: 13904-13911.

Stoneham ET, Sanders EM, Sanyal M, Dumas TC. 2010. Rules of engagement: factors that regulate activity-dependent synaptic plasticity during neural network development. Biol Bull 219: 81-99.

Tan HM, Wills TJ, Cacucci F. 2016. The development of spatial and memory circuits in the rat. Wiley Interdiscip Rev Cogn Sci 125: 159-167.

Travaglia A, Bisaz R, Sweet ES, Blitzer RD, Alberini CM. 2016. Infantile amnesia reflects a developmental critical period for hippocampal learning. Nat Neurosci 19: 1225-1233.

Wallace DG, Gorny B, Whishaw IQ. 2002. Rats can track odors, other rats, and themselves: implications for the study of spatial behavior. Behav Brain Res 131: 185-192.

Warburton EC, Brown MW. 2015. Neural circuitry for rat recognition memory. Behav Brain Res 285: 131-139.

Wills TJ, Cacucci F, Burgess N, O'Keefe J. 2010. Development of the hippocampal cognitive map in preweanling rats. Science 328: 1573-1576.

Received July 20, 2017; accepted in revised form December 22, 2017. 


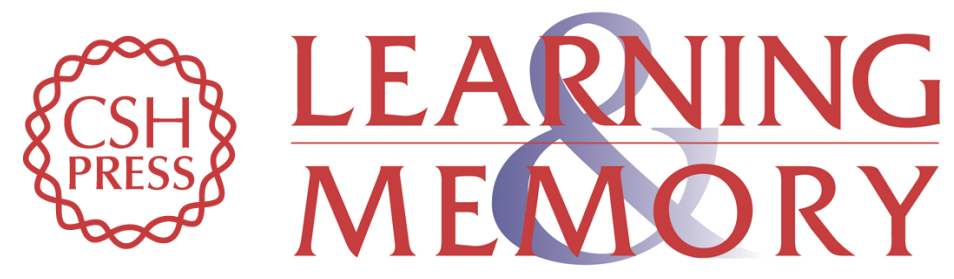

\section{A Barnes maze for juvenile rats delineates the emergence of spatial navigation ability}

Daniel G. McHail, Nazanin Valibeigi and Theodore C. Dumas

Learn. Mem. 2018, 25:

Access the most recent version at doi:10.1101/Im.046300.117

\section{Supplemental http://learnmem.cshlp.org/content/suppl/2018/02/09/25.3.138.DC1 Material}

References This article cites 49 articles, 5 of which can be accessed free at: http://learnmem.cshlp.org/content/25/3/138.full.html\#ref-list-1

Creative This article is distributed exclusively by Cold Spring Harbor Laboratory Press for the Commons first 12 months after the full-issue publication date (see

License http://learnmem.cshlp.org/site/misc/terms.xhtml). After 12 months, it is available under a Creative Commons License (Attribution-NonCommercial 4.0 International), as described at http://creativecommons.org/licenses/by-nc/4.0/.

Email Alerting Receive free email alerts when new articles cite this article - sign up in the box at the Service top right corner of the article or click here. 